\title{
Analyse d'un programme de développement des productions fruitières en Polynésie française
}

Yves BeRTIN*

Cirad, département Flhor, TA 50 / PS 4, Boulevard de la Lironde, 34398 Montpellier Cedex 5, France

yves.bertin@cirad.fr
* Correspondance et tirés à part

Fruits, 2006, vol. 61, p. 9-23 (C) 2006 Cirad/EDP Sciences All rights reserved DOI: 10.1051/fruits:2006002

RESUMEN EsPañol, p. 23

\section{Analysis of a development program of the fruit-bearing trees in French Polynesia.}

Abstract - Context and project presentation. After a short history and general view of fruit-bearing tree crops in French Polynesia, the functioning and operations programed over 3 years of a support project carried out from 2002 to 2005 are presented. Realization. The five principal topics of action are successively described: improvement of the vegetable material; accompanying research; training, extension and farmer organization and training: topic supplemented by the drafting of technical documents; evaluation missions and training courses; quality approach and professional organization, supplemented by a part on commodity development by processing. Results and inventoried resources are analyzed. Discussion and conclusion. Benefits and disadvantages encountered in the project realization are discussed and make it possible to propose some improvements in the implementation of similar projects.

French Polynesia / fruit growing / agricultural development / surveys / plant introduction / choice of species
Analyse d'un programme de développement des productions fruitières en Polynésie française.

Résumé - Contexte et présentation du projet. Après un bref historique et un aperçu général de l'arboriculture fruitière en Polynésie française, le fonctionnement et les opérations programmées sur 3 ans d'un projet d'appui mené de 2002 à 2005 sont présentés. Réalisation. Les cinq thèmes principaux d'intervention sont successivement décrits : amélioration du matériel végétal ; recherches d'accompagnement ; formation, vulgarisation et encadrement : volet complété par la rédaction de documents techniques; missions d'appui et stages; démarche qualité et organisation de la profession, complété par un volet de valorisation des produits par transformation. Les résultats et les moyens utilisés sont analysés. Discussion et conclusion. Les avantages et inconvénients rencontrés dans la réalisation du projet sont discutés et permettent de proposer quelques améliorations dans la mise en œuvre de projets analogues.

Polynésie française / culture fruitière / développement agricole / enquête / introduction de plantes / choix des espèces 
Figure 1.

Présentation des cinq archipels de la Polynésie française.

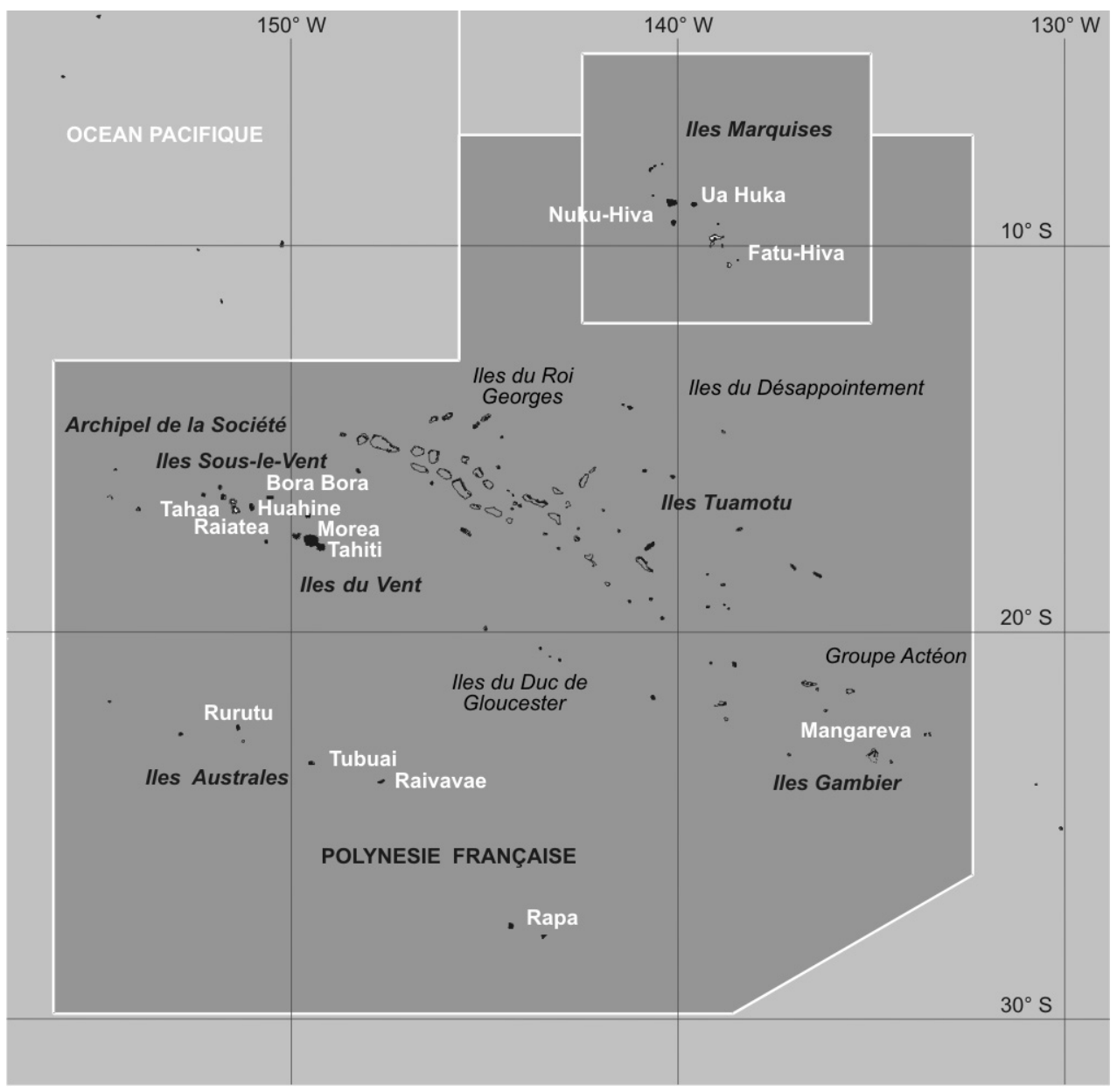

\section{Introduction}

\subsection{Situation géographique}

La Polynésie française, d'une superficie totale comparable à celle de l'Europe, comprend 118 îles regroupées en cinq archipels : les îles Marquises, les Tuamotu, les îles de la Société, les Gambier et l'archipel des Australes (figure 1). Ces îles sont très isolées dans le Pacifique Sud, entre $7^{\circ} 50^{\prime}$ et $27^{\circ} 36^{\prime}$ de latitude $S$ et entre $154^{\circ} 40^{\prime}$ et $134^{\circ} 28^{\prime}$ de longitude $\mathrm{O}$. Ce sont en majorité des atolls coralliens très plats ou des îles volcaniques accidentées atteignant parfois des altitudes supérieures à $1000 \mathrm{~m}$.

D'un archipel à l'autre, il existe de grandes variations climatiques : climat de type tropical humide, voire équatorial, aux îles
Marquises, jusqu'au climat subtropical aux îles Australes. Cette grande diversité permet d'envisager la culture de très nombreuses espèces fruitières lorsque le sol, parfois très calcaire, ou la disponibilité en eau ne constitue pas un facteur limitant.

\subsection{Début des productions fruitières en Polynésie}

À l'instigation du maire d'Ua Huka (île de l'archipel des Marquises) à la recherche d'activités agricoles pour diversifier les productions de l'île, une première collection d'arbres fruitiers tropicaux divers fut plantée dans les années 1990. À partir de 1994, l'implication d'un ingénieur du Centre de coopération internationale en recherche agronomique pour le développement (Cirad) permit de lancer une opération de plus 
grande envergure. Une collection d'agrumes comportant 116 variétés provenant de la station Inra-Cirad de San-Giuliano en Corse fut alors mise en place avec l'appui du Service local de développement rural (SDR) du ministère de l'Agriculture qui mit à la disposition de cette opération du personnel et une parcelle d'expérimentation.

Au cours des années 1995 à 1997, le conservatoire variétal fut un peu négligé par manque de moyens et de personnel. Cependant, en 1995, une expertise du Cirad [1] avait démontré le grand intérêt du matériel végétal implanté et préconisé l'installation de deux autres parcelles, plus modestes, l'une aux Australes et l'autre à Raïatea (îles Sous-le-Vent). Cette mission avait jeté aussi les bases d'une stratégie de développement de l'agrumiculture en Polynésie.

Des conservatoires variétaux d'agrumes furent par suite mis en place par le SDR, mais, comme à l'île d'Ua Huka, leur suivi ne fut pas suffisant. Une convention fut alors signée entre le SDR et la mairie de Ua Huka pour régir les rôles respectifs des différentes institutions afin d'exploiter au mieux le matériel végétal implanté en collection. En 1998, une nouvelle évaluation, par deux experts du Cirad [2], des agrumes mis en place suggéra que ces premières expérimentations soient prolongées par un développement global de la filière " fruits ", assorti de certaines mesures d'accompagnement, dont un volet important pour la formation d'un personnel adapté au suivi des productions fruitières.

À partir de l'année 2000, la nécessité de faire appel à un agent spécialisé pour suivre l'ensemble du programme fruitier lancé en Polynésie se fit évidente. Les maires des six îles de l'archipel des Marquises rédigèrent une demande de financement conjointe pour un projet d'assistance aux producteurs de fruits de la région. Avec l'aide du Cirad, ce projet établi pour une période de trois ans fut chiffré. Son évaluation administrative par le Territoire déboucha alors sur un élargissement de l'opération à tous les archipels de Polynésie française. Un programme fut établi en décembre 2001 définissant cinq thèmes d'action principaux : amélioration de la production de matériel végétal fruitier ; recher- ches d'accompagnement visant à valoriser particulièrement les conservatoires variétaux ; formation, vulgarisation et encadrement des agents du SDR et des agriculteurs ; organisation de missions d'appui scientifique et technique sur des sujets bien identifiés ; amélioration de la qualité, commercialisation et valorisation des produits. Ces volets ont été complétés par la rédaction de documents techniques.

L'opération débuta en mai 2002 pour une période de trois ans avec l'affectation d'un ingénieur agronome du Cirad nommé responsable de la réalisation du projet.

\subsection{Organisation du projet et programme retenu}

L'île d'Hiva Oa, offrant une situation centrale dans l'archipel des îles Marquises et possédant les plus grandes possibilités de développement des arbres fruitiers, a été retenue comme base géographique du programme.

Un ingénieur responsable de la filière "fruits " au service de développement rural fut identifié pour faire la liaison avec le responsable du projet nommé par le Cirad. Après formation auprès de ce responsable, l'agent du SDR a été chargé de rechercher des financements et de mettre en place les opérations envisagées par le projet pour le développement de la filière fruit.

Parallèlement à ce tandem d'agents CiradSDR, un " comité technique de suivi et de programmation " a été constitué. À la faveur de deux réunions par an, ce comité ${ }^{1}$ devait faire le point sur l'avancement du projet et, éventuellement, modifier ou réorienter le programme retenu. Un planning des opérations fut établi en cohérence avec les cinq thèmes d'action définis pour le programme (tableau I).

${ }^{1}$ Ce comité a été composé du ministre de l'Agriculture, des maires de Rurutu aux Australes et de Ua Huka aux Marquises, ainsi que du chef de service du SDR (assisté de responsables de services), du directeur régional du Cirad en Polynésie et de l'agent responsable du projet. 
Tableau I.

Planning des opérations prévues par les thèmes retenus pour la réalisation d'un projet de développement des productions fruitières en Polynésie française (2002 à 2005).

Désignation

Nombre de mois après le début du projet

$\begin{array}{llllllllllllllllll}2 & 4 & 6 & 8 & 10 & 12 & 14 & 16 & 18 & 20 & 22 & 24 & 26 & 28 & 30 & 32 & 34 & 36\end{array}$

1 - Recherche d'accompagnement

Exploitation des données des conservatoires

Création d'une banque de données sur les vergers de référence

Choix et rédaction de fiches variétales

Études des possibilités de produits spécifiques

Études des possibilités d'étalement des

productions

Comportement de nouveaux porte-greffes

Adaptation des programmes de fertilisation

Définition des meilleurs stades de récolte

Veille sanitaire en relation avec la DPV

Programme de prémunition des agrumes

2 - Amélioration du matériel végétal Définition des modalités de production de semences

Diffusion du matériel issu des conservatoires

Constitution de parcs à bois complémentaires

Aide à la mise en place d'une certification (DPV)

Formation des pépiniéristes

Établissement des coûts de production de plants

3 - Commercialisation, valorisation des produits

Analyse des pratiques et des freins

Établissement des coûts de production

Amélioration, conservation et transport

Étude d'emballages appropriés

Levée des verrous sanitaires : techniques,

traitement

Identification de produits à transformer

Innovation en technologie agroalimentaire (LTA)

4 - Formation, vulgarisation, encadrement

Création d'un fond de documentation

Rédaction de notes techniques

Participation à la formation des agents de terrain

Diffusion de fiches variétales

Informations fruitières par les médias

Formation de groupes d'agriculteurs

5 - Missions d'appui scientifique

État des lieux en virologie et suivi de la tristeza

État des lieux en entomologie

Étude des systèmes d'activités et de production

DPV : Direction de la protection des végétaux ; LTA : laboratoire de technologie alimentaire. 


\section{4. État des productions fruitières en Polynésie lors du lancement du projet}

Au moment du lancement du projet de développement fruitier en Polynésie française, le secteur agricole représentait la troisième ressource financière après le tourisme et l'exploitation de la perle ${ }^{2}$. Les principales spéculations agricoles étaient, par ordre décroissant d'importance, les produits marâ̂chers, les productions animales, la floriculture, les fruits et le coprah. Cependant, des productions marginales, comme les cultures vivrières, le bois, la vanille, le nono (Morinda citrifolia) ou les produits végétaux exploités par l'artisanat, pouvaient être notées (figure 2).

Les cultures fruitières se concentraient essentiellement sur deux archipels qui totalisaient plus de $95 \%$ de la production totale : îles de la Société avec les îles du Vent (Tahiti, Moorea) et les îles Sous-le-Vent (Raïatea, Tahaa, Bora Bora et Huahine), et les îles Marquises avec $19 \%$.

Les statistiques agricoles de 2003 [3] rapportent 1268 ha de superficies plantées en cultures fruitières pour une production commercialisée de 2938 t (tableau II), dont ne font pas partie les melons, pastèques et ananas classés dans d'autres filières. Ces chiffres traduisent une mauvaise répartition de la production sur le Territoire et surtout de faibles volumes commercialisés. Comme ils ne prennent en compte que les circuits commerciaux traditionnels (marchés, grandes surfaces et grossistes), ils excluent les ventes directes et l'autoconsommation qui constitueraient près de $80 \%$ de la consommation des ménages.

La répartition des productions fruitières produites et commercialisées en 2003 dans les archipels de Polynésie mettait en évidence la dominance des productions de Tahiti et de Moorea par rapport à celles des Tuamotu et des Gambier. Ces îles en très grand nombre sont pour la plupart des atolls coralliens peu propices à la culture des arbres fruitiers. Cependant, certaines îles des Gambier, de nature différente, auraient un potentiel intéressant pour la culture du

\footnotetext{
${ }^{2}$ Selon le site www.presidence.pf.
}

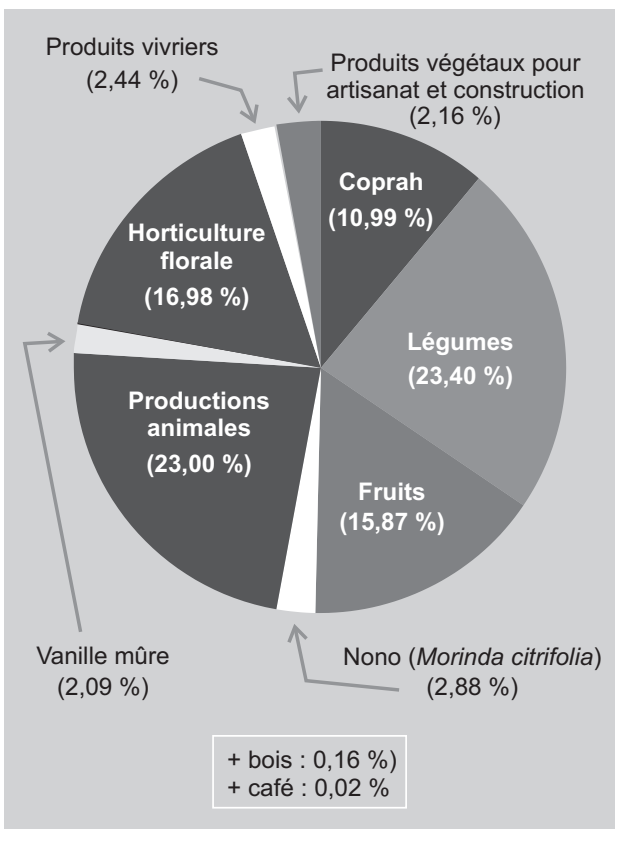

Figure 2.

Répartitions des principales spéculations agricoles de la Polynésie française (en \% de la valeur commerciale).

litchi. Les agrumes et la banane représentent la plus grande part (plus de $80 \%$ ) des fruits commercialisés.

\section{La réalisation du programme de développement fruitier}

Pour réaliser le programme de développement fruitier en Polynésie française, les cinq thèmes prévus par le projet ont été abordés.

\subsection{Amélioration de la production de matériel végétal}

Un premier état des lieux effectué dès le début du projet sur les douze principales pépinières gérées par le SDR dans les différents archipels a révélé, d'une façon générale, que la technicité des pépiniéristes était insuffisante : mesures de contrôle sanitaire le plus souvent inexistantes, origine des greffons non contrôlée et diffusion de plants d'agrumes issus de semis ou de marcottes apte à menacer l'avenir des plantations du fait de risques de tristeza et d'autres maladies virales ou cryptogamiques. Enfin, chez les pépiniéristes, il apparaissait un déficit chronique en matériel et équipements 
Tableau II.

Part commercialisée des productions de fruits en Polynésie française présentées par archipel et par espèce fruitière (2003) [3].

\begin{tabular}{|c|c|c|c|c|c|c|c|c|}
\hline \multirow[t]{2}{*}{ Archipel } & Avocat & Banane & Banane séchée & Citron (limes) & Corossol & Kava & Mandarine & Mangue \\
\hline & \multicolumn{8}{|c|}{$(\mathrm{t})$} \\
\hline Îles du Vent & 32,0 & 771,7 & 1,3 & 411,3 & 0,8 & - & 196,6 & 62,8 \\
\hline Îles Sous-le-Vent & 4,0 & 15,8 & - & 7,5 & 0,1 & - & 0,7 & 0,4 \\
\hline Marquises & 0,1 & 18,2 & 0,6 & 159,5 & - & 0,1 & 0,5 & 26,5 \\
\hline Australes & 0,3 & 7,6 & - & 12,2 & - & - & - & - \\
\hline Tuamotu-Gambier & - & 0,6 & - & 0,9 & - & - & - & - \\
\hline Polynésie 2003 & 36,4 & 813,9 & 1,9 & 591,4 & 0,9 & 0,1 & 197,8 & 89,7 \\
\hline
\end{tabular}

\begin{tabular}{|c|c|c|c|c|c|c|c|c|c|c|}
\hline \multirow[t]{2}{*}{ Archipel } & Mape & Orange & Pamplemousse & Papaye & Litchis & $\begin{array}{l}\text { Pomme } \\
\text { Cannelle }\end{array}$ & $\begin{array}{l}\text { Pomme Etoile } \\
\text { (Caïmite) }\end{array}$ & Quenette & Ramboutan & Autres \\
\hline & \multicolumn{10}{|c|}{$(\mathrm{t})$} \\
\hline Îles du Vent & 1,0 & 402,6 & 455,9 & 239,2 & 2,9 & 0,6 & 0,5 & 0,6 & 11,9 & 1,4 \\
\hline Îles Sous-le-Vent & 1,4 & 0,6 & 8,4 & 13,5 & - & - & 0,1 & - & 5,4 & 0,1 \\
\hline Marquises & 2,1 & 6,1 & 24,5 & 3,4 & - & - & - & - & 1,0 & 0,6 \\
\hline Australes & 0,3 & 0,4 & 1,9 & 0,9 & 17,0 & 0,3 & - & - & - & - \\
\hline Tuamotu-Gambier & - & - & - & - & 2,5 & - & - & - & - & - \\
\hline Polynésie 2003 & 4,8 & 409,7 & 490,7 & 257,0 & 22,4 & 0,9 & 0,6 & 0,6 & 18,3 & 2,1 \\
\hline
\end{tabular}

\begin{tabular}{lcc} 
Archipel & $\begin{array}{c}\text { Production totale commercialisée } \\
\text { en } 2003 \\
(\mathrm{t})\end{array}$ & $\begin{array}{c}\text { Répartition selon les } \\
\text { archipels } \\
(\%)\end{array}$ \\
\hline Îles du Vent & 2592,8 & 88,23 \\
îles Sous-le-Vent & 57,9 & 1,97 \\
Marquises & 243,1 & 8,27 \\
Australes & 40,8 & 1,39 \\
Tuamotu-Gambier & 4,0 & 0,14 \\
Polynésie 2003 & 2938,6 & 100
\end{tabular}

ble et indemne de virus en Polynésie était le conservatoire variétal de l'île d'Ua Huka. Cependant, l'approvisionnement des pépinières effectué à partir de cette source de greffons n'a pas donné que des résultats satisfaisants ; ainsi, l'éloignement de la plus importante pépinière située à Tahiti occasionnait des coûts et une organisation spécifique des transports aériens peu propices au développement des plantations. La mise en place, pour cette unité, d'un parc à bois sous serre insect-proof a alors été suggérée. appropriés. Par ailleurs, les plants étant Devenue autonome, cette installation devait cédés par le SDR aux agriculteurs à un prix permettre, compte tenu de la situation ceninférieur au coût réel, il était difficile aux trale de Tahiti, de fournir également des pépiniéristes privés de se lancer dans la pro- greffons indemnes de tristeza à d'autres îles duction de plants fruitiers à un prix rému- avoisinantes. À la fin du projet, l'installation nérateur.

Ces différents constats ont conduit à préconiser différentes mesures :

- Élimination de tous les plants d'agrumes issus de semis et de marcotte et forte recommandation aux greffeurs de n'utiliser que des sources de greffons fiables. Dans un premier temps, la seule origine vraiment fiade ce parc à bois sous serre était réalisée et les premiers plants du parc à bois étaient en cours de culture.

- Mise en place d'équipements spécifiques afin de rationaliser et d'améliorer la qualité des plants en pépinière : tables de semis, irrigation, dalles cimentées, abris de greffage, abris pour les mélanges terreux et adoption 
d'un système d'étiquetage systématique par bloc et par plant. Progressivement les différentes pépinières ont acquis ces équipements et, à la fin du projet, la majorité d'entre elles était presque totalement équipée.

- Formation des agents pépiniéristes, action décisive dans la réussite du programme de développement. Ce volet a représenté un travail permanent pendant toute la durée du projet et sera détaillé ultérieurement.

- Établissement du prix de revient des plants dans les cinq pépinières principales. Cela a mis en évidence des écarts importants entre les pépinières et a permis de corriger les imperfections liées le plus souvent à une mauvaise utilisation de la main-d'œuvre. Dans tous les cas, même après rationalisation de la pépinière, le prix de revient s'est révélé supérieur au prix de vente (identique depuis 1974). Les évènements politiques de 2004 et début 2005 n'ont pas permis d'obtenir la modification du prix des plants qui constitue un point sensible politiquement.

- Mise en place d'un processus de production de plants " certifiés ". Une telle mesure implique que les pépiniéristes aient un bon niveau technique et que tous les éléments de ce processus puissent être maîtrisés dans toutes les pépinières. À l'issue du projet, ce n'était pas encore le cas. Les formations avaient bien eu lieu, mais la certification proprement dite n'avait pas encore débuté.

Globalement les objectifs liés à l'amélioration, en pépinière, de la production de matériel végétal en qualité et en quantité ont été atteint au cours du projet. En 2005, la technicité des pépinières était tout à fait satisfaisante pour une grande majorité d'entre elles. Si certaines accusaient un retard, d'autres produisaient des plants d'une qualité irréprochable tant sur leur conformation que sur leur état sanitaire. À cette date, environ 20000 plants $a n^{-1}$ étaient produits par les pépinières de Polynésie, ce qui représentait un potentiel de plantation de l'ordre de 100 ha.

\subsection{Recherches d'accompagnement}

\subsubsection{Enquête tristeza}

La maladie de la tristeza, grave maladie des agrumes, avait été identifiée dans les plan- tations de limes des îles de la Société au cours des années 1970. Cependant, des prospections effectuées dans les autres archipels n'avaient pas permis de la détecter. Il convenait de confirmer ce point par prélèvement systématique d'échantillons dans les îles visitées et surtout de veiller à ce que le conservatoire variétal d'Ua Huka reste indemne. Tous les contrôles de la maladie effectués au cours du projet se sont révélés négatifs dans les conservatoires des îles Marquises et des Australes, ainsi que dans les différents archipels Gambier (Mangareva) et Australes (Rurutu, Tubuaï, Raïvavae et Rapa). Cependant, les Tuamotu n'ayant pas encore été prospectées à la fin du projet, il serait nécessaire de les contrôler également vis-à-vis de cette menace de tristeza.

\subsection{2. Évaluation des variétés d'agrumes}

L'existence de trois conservatoires de variétés d'agrumes aux îles Marquises (116 variétés), Australes (26 variétés) et îles Sous-leVent à Raïatea (15 variétés) s'est révélée propice à une étude multilocale du comportement des différentes variétés sous différentes conditions écologiques. Pour cela, des observations systématiques des parcelles ont été effectuées et des fiches descriptives illustrées par des photos de fruits ont été établies pour toutes les variétés présentes à Ua Huka (figure 3). Des équipements de laboratoire complémentaires, financés par le SDR, ont pu être acquis pour affiner les analyses de fruits déjà effectuées et compléter ces fiches. Ce complément de travail a débuté dans la dernière année du programme avec des agents responsables préalablement formés pour ces observations.

L'exploitation des résultats obtenus a permis d'autre part d'identifier les variétés les mieux adaptées et d'éditer un catalogue complet décrivant chacune d'elles. Au total 27 variétés réparties parmi les principales espèces de Citrus ont été retenues pour une exploitation possible dans les différents archipels. Une diffusion systématique de ces variétés sélectionnées devrait être validée par les techniciens du SDR et par les agriculteurs à partir de tests de dégustation de fruits.

En complément de ces travaux de sélection, une évaluation des collections de 
Figure 3.

Exemple de fiche variétale (format $\mathrm{A} 4$ ) rédigée après évaluation de la collection d'agrumes mise en place aux îles Marquises (Polynésie françaises).
Figure 4.

Couverture du catalogue des variétés (format $A 4$ ) diffusées par le service de développement rural en Polynésie française, présentant les variétés des conservatoires d'Ua Huka et de Rurutu.
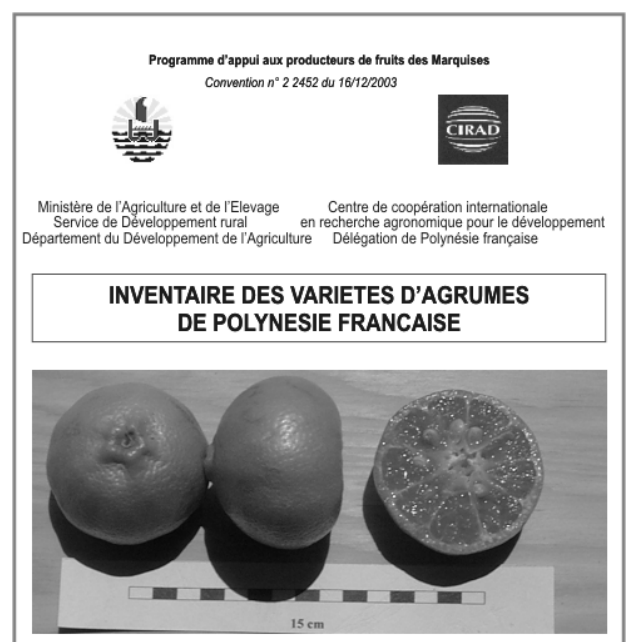

place, en Polynésie, d'un véritable Centre de ressource biologique (CRB) d'importance internationale, qui pourrait bénéficier à toute la région du Pacifique Sud caractérisée par des conditions sanitaires exceptionnelles. D'ores et déjà les collections polynésiennes ont pu s'enrichir de 69 variétés supplémentaires et de 14 nouveaux porte-greffes rapportés du conservatoire de Corse (figure 4).

\subsection{Formations, vulgarisation et encadrement}

Les opérations de formation et d'encadrement des agents du service de développement rural, du technicien pépiniériste jusqu'à l'ingénieur, ainsi que celles de vulgarisation, réservées aux agriculteurs, ont constitué le travail le plus important du chef de projet.

\subsubsection{Formations spécifiques locales}

Pendant les 3 années de réalisation du projet, 35 pépiniéristes ont été formés aux techniques de multiplication de plants sains, 25 techniciens ont appris à appliquer la lutte raisonnée contre les ravageurs des arbres fruitiers et 28 agents du SDR ont reçu une formation générale en arboriculture fruitière. D'autres formations plus ponctuelles n'ont pu être assurées du fait de la conjoncture politique des années 2004 et 2005.

Variétés des Conservatoires de Ua Huka et de Rurutu

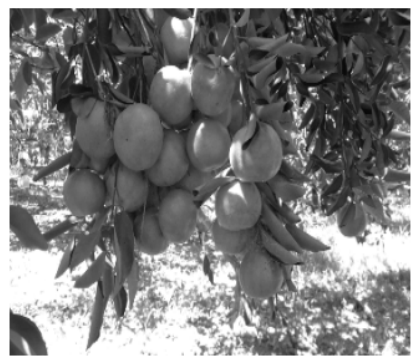

CATALOGUE DES VARIETES DIFFUSEES PAR LE SDR EN POLYNESIE

Polynésie a été effectuée par deux spécialistes agrumes du conservatoire de San Giuliano (inra-Cirad, Corse). Il s'en est suivi l'établissement d'un véritable partenariat scientifique entre les deux structures et les experts consultés ont proposé la mise en

\subsubsection{Formations reçues en dehors de la Polynésie}

Le programme de production fruitière entrepris dans les archipels de la Polynésie française a permis à un ingénieur du SDR ainsi qu'à un technicien responsable du conservatoire d'Ua Huka de faire un séjour en Corse sur la station Inra-Cirad pour prendre connaissance du fonctionnement de ce conservatoire de référence. Par ailleurs, quatre pépiniéristes ont pu se rendre à l'institut agronomique de Nouvelle-Calédonie (IAC) afin d'établir des relations de partenariat avec cet autre territoire français. En plus de rompre l'isolement insulaire des participants à ces formations, de tels déplacements ont permis de redonner à ces professionnels une meilleure implication dans l'exercice de leurs activités. 


\subsubsection{Vulgarisation à destination des agriculteurs}

Les expertises effectuées dans les archipels de Polynésie ont montré que seule l'île de Tahiti disposait d'arboriculteurs possédant une technicité appropriée. Dans la majorité des autres îles, de nombreuses notions horticoles essentielles ont semblé méconnues : précautions à prendre lors de la plantation, taille adéquate des arbres, apports raisonnés d'engrais, identification et contrôle des ravageurs et maladies, etc. Par des visites régulières de plantations, un important travail de vulgarisation a permis au chef de projet détaché par le Cirad de diffuser systématiquement, avec l'aide des techniciens de terrain présents sur les îles visitées, des notions de bases sur l'entretien des vergers et, notamment, sur la plantation des arbres fruitiers, leur taille et la connaissance des variétés, accompagnée de dégustations.

Pour atteindre un public plus large et sensibiliser la population à certaines techniques arboricoles, quelques émissions ont été organisées sur "Radio Marquises " sous forme de questions-réponses.

\subsubsection{Formation de l'ingénieur responsable de la filière "fruits" au SDR}

Au moment du lancement du projet pour le développement des productions fruitières en Polynésie, l'ingénieur responsable de la filière " fruits " au service de développement rural débutait ses fonctions. En travaillant étroitement avec le chef de projet spécialiste d'arboriculture fruitière qu'il accompagnait dans toutes les visites de plantations, il lui a été possible de se perfectionner techniquement. Cette formation sur le terrain a été complétée par un voyage d'étude en Corse et à l'île de la Réunion où il a pu rencontrer de nombreux spécialistes du Cirad et de l'Inra avec lesquels il a engagé des partenariats.

\subsubsection{Rédaction de documents techniques}

En appui aux actions de formation et de vulgarisation, la rédaction de documents techniques à mettre à la disposition des différents agents de la filière "fruits " est rapidement apparue comme indispensable au dévelop-

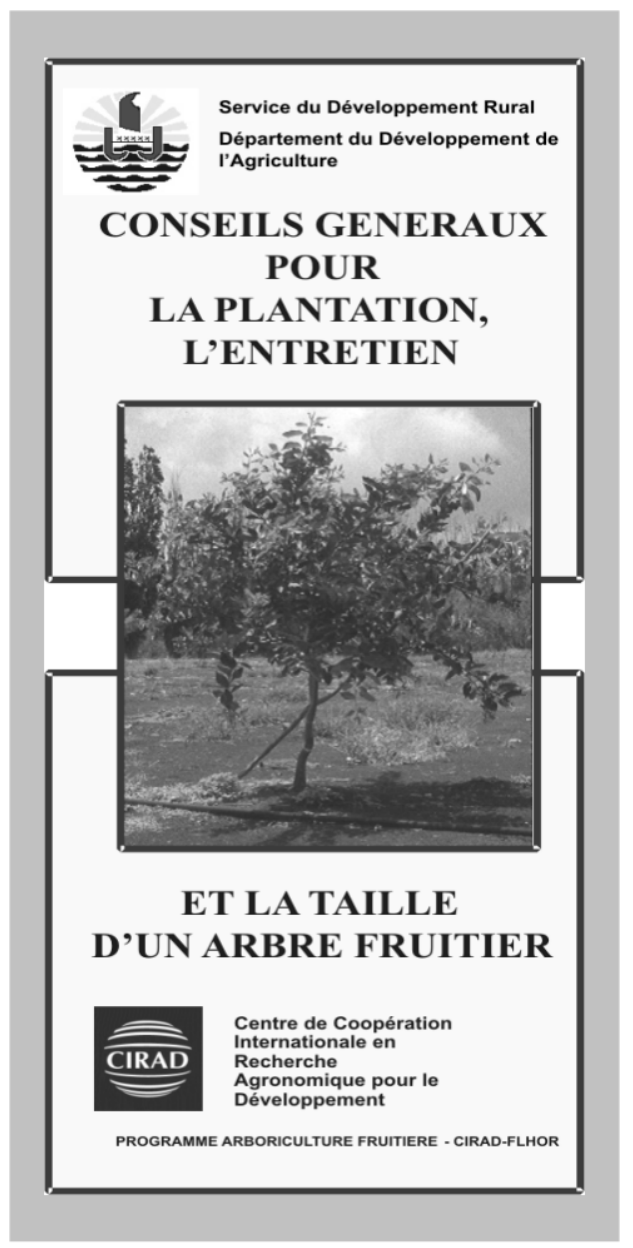

Figure 5.

Dépliant de trois volets (format A4 déplié), distribué aux agriculteurs en Polynésie française permettant de vulgariser des techniques expérimentées pour l'arboriculture fruitière. pement des activités entreprises et à la mise en commun d'un langage unique pour tous les praticiens de la culture de l'arbre fruitier. Ainsi différents documents ont été publiés parmi lesquels un dépliant ${ }^{3}$ (figure 5), en français, tahitien et marquisien, sur la plantation, l'entretien et la taille des arbres fruitiers a été diffusé en 3000 exemplaires ; neuf fiches techniques ${ }^{3}$ dédiées spécifiquement aux agrumes, banane, mangue, papaye, litchi, vigne, fruit de la passion (figure O), avocat et goyave ont également été réalisées ; ces documents ont été complétés par une note plus spécifique ${ }^{3}$ sur la récolte et l'aprèsrécolte des agrumes. Les aspects économiques de la production ont été pris en compte

3 Ces documents sont disponibles auprès du Service de développement rural de Papeete, BP 100, 98713 Papeete, Tahiti. 
Figure 6.

Exemple de fiche technique (format A4) rédigée en appui au volet de formation des agriculteurs en Polynésie française.

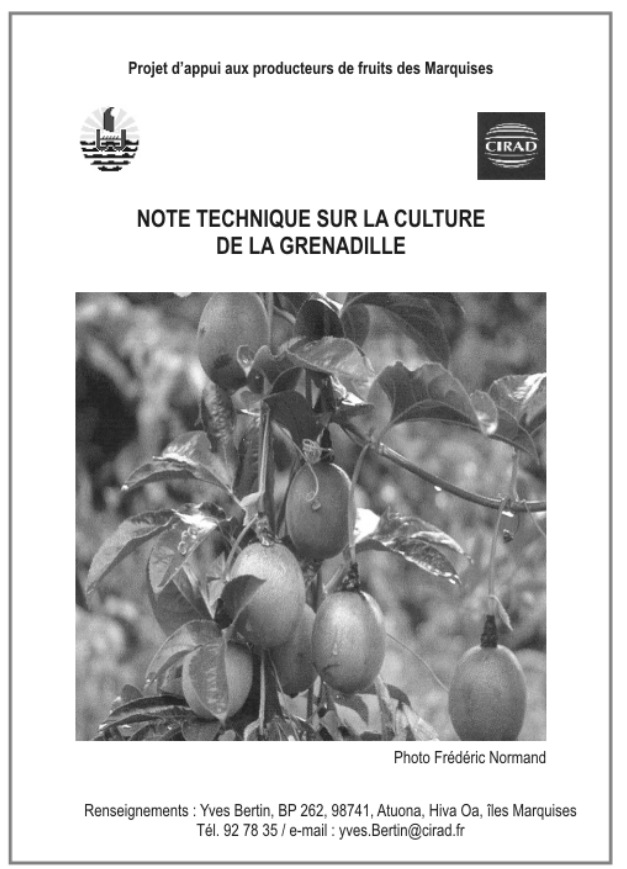

pour la rédaction d'un document ${ }^{3}$ proposant un cahier des charges minimum pour la commercialisation des agrumes sur le territoire de Polynésie française et d'une fiche d'établissement ${ }^{3}$ des coûts de production pour différentes cultures fruitières. Enfin, une note présentant un itinéraire technique pour une culture biologique des agrumes a été mise à la disposition des agriculteurs intéressés.

\subsection{Organisation de missions de scientifiques et de stages}

Dès la conception du projet, des missions de scientifiques et des stages de longue durée ont été prévus en appui au chef de projet. Ces interventions axées sur des points très spécifiques ont permis d'approfondir des problèmes ne relevant pas de compétences locales.

\subsubsection{Interventions de scientifiques en Polynésie}

Une série d'expertises focalisées sur les problèmes sanitaires ou sur la conservation des ressources génétiques a conduit à accueillir en Polynésie :
- la mission d'un entomologiste du Cirad [4] qui a permis d'actualiser l'inventaire des ravageurs des cultures fruitières et de définir les meilleurs moyens de lutte à utiliser ;

- une prospection pathologique et virologique [5] effectuée conjointement par le Cirad et le SDR qui a vérifié l'état sanitaire des principaux archipels vis-à-vis en particulier des maladies graves des agrumes (tristeza, chancre citrique et Huanglongbing) : les îles de la Société ont été confirmées comme inféodées à la tristeza, alors que les archipels des Australes et des Marquises ont été trouvées indemnes de ces maladies ;

- un spécialiste de la lutte raisonnée en verger de la chambre d'agriculture de l'île de la Réunion, qui a dispensé aux techniciens du SDR une formation très spécialisée sur le sujet ;

- une évaluation des conservatoires variétaux polynésiens par deux ingénieurs de la station agrumicole de Corse (SRA-Cirad) qui a abouti à la définition d'une stratégie de gestion des conservatoires existants et au lancement d'une collaboration scientifique sur les ressources biologiques agrumes [6].

\subsection{2. Études spécifiques}

Plusieurs études effectuées dans le cadre de formation diplômante ont été menées durant la réalisation du projet ; elles ont donné lieu à la publication de rapports :

- le fonctionnement des exploitations agricoles aux îles Marquises a été étudié par Talem [7] qui a montré la part importante de la pluri-activité des exploitants et le poids non négligeable des échanges informels du fait que $40 \%$ des revenus de ces exploitations échappent au commerce traditionnel ;

- à Tahiti et Moorea où l'arboriculture est la plus développée, de la Torre [8] s'est intéressée à la typologie des exploitations fruitières. Ce stage a permis d'effectuer une photographie des exploitations et de démarrer un fichier exhaustif des plantations fruitières des îles concernées par l'étude ;

- à la demande d'un industriel de Moorea souhaitant valoriser les gisements naturels de mangues et de goyaves des îles Marquises tout en s'affranchissant partiellement des importations de pulpe, Faucon [9] a effectué 
l'inventaire des variétés de manguiers dans l'archipel et étudié les possibilités de transformation des productions de mangues et de goyaves. Si techniquement les variétés répertoriées conviennent bien, il en ressort que les problèmes de ramassage, de coût de transport et de stockage doivent être résolus avant de développer l'étape de la transformation.

\subsection{Amélioration de la qualité et organisation de la profession, valorisation des produits}

\subsubsection{Amélioration de la qualité et organisation de la profession}

L'approvisionnement en fruits des grandes surfaces de Tahiti à partir des productions locales n'est pas sans problème. Les agriculteurs peu habitués à une démarche "qualité " ont des difficultés à écouler leur production vers ces grandes surfaces qui exigent des fruits irréprochables et qui pratiquent des réductions de prix importantes dès qu'il y a du tri à faire. D'autre part un système de régulation des importations en période de production oblige les commerçants à passer par la production locale.

Tant que les volumes commercialisés resteront déficitaires par rapport à la demande du marché local, les agriculteurs aidés par le système de régulation parviendront à maintenir des prix élevés ; cependant, les productions étant en augmentation, les agriculteurs commencent à s'intéresser à la qualité. Une démarche " qualité "a donc été élaborée et leur a été proposée ; cela étant, pour le moment, ils refusent de l'appliquer de crainte d'une législation trop contraignante et d'une baisse de leur chiffre d'affaires.

Dans un tel marché de pénurie, la structuration de la profession se fait très lentement, mais quelques groupements se sont cependant créés avec l'appui du projet fruitier : trois îles (Tahaa, Moorea et Hiva Oa) disposent désormais de leur syndicat ou de leur association de producteurs.

Une large étude pour le développement des archipels, financée par le Fonds européen de développement, a été conduite pendant près de trois ans par un bureau d'étude. Le projet fruitier a collaboré avec le SDR à l'élaboration du programme de développement de la filière "fruits ". Ces actions devraient aboutir à la création d'un centre de regroupement des productions marâichères et fruitières sur l'île de Tahiti et à la mise en place de petites structures de collecte des fruits dans les archipels.

\subsubsection{La valorisation des produits : le secteur de la transformation}

L'accompagnement par le projet fruitier du secteur de la transformation a débouché sur quelques projets en cours de réalisation ou d'élaboration :

- extraction et congélation de la purée de goyave de Chine dans une unité de transformation de l'île de Rapa aux îles Australes ( $1 \mathrm{t}$ produite en 2004) ;

- mise en route d'un atelier de transformation des fruits à Ua Huka : congélation de pulpe de mangue et de goyave, fabrication de confitures et fruits confits

- production de jus de citron, lait et eau de coco et purée de fruits (corossol) prévu par un projet de développement privé en cours de développement à Nuku Hiva ;

- étude de la qualité industrielle des mangues des îles Marquises et de l'amertume des jus de pamplemousses destinée à appuyer l'usine de jus de fruits de Moorea.

\section{Financement du projet}

Le projet d'appui aux producteurs de fruits polynésiens a pu se dérouler dans de bonnes conditions de mai 2002 à mai 2005, grâce à un financement mis en œuvre par le territoire de la Polynésie française. Au cours de ces trois années, les différents archipels ont été visités à l'occasion de 36 déplacements effectués par le chef de projet accompagné de l'ingénieur du Service de développement rural dans une vingtaine d'îles, chacune d'elles ayant donné lieu à une ou plusieurs visites. Les moyens accordés au projet ont donc permis une présence permanente de professionnels de l'arboriculture fruitière auprès des acteurs du développement.

Parallèlement des moyens important ont pu être mobilisés par le SDR pour réaliser 
Tableau III.

Récapitulatif des coûts, détaillés par action, correspondant au financement du projet d'appui aux producteurs de fruits polynésiens.

( $1 € \approx 120 \mathrm{~F}$ Pacifique).

1. Financement de la Polynésie française : $96600 \times 10^{3}$ F CFP TTC.

\begin{tabular}{|c|c|}
\hline Opérations & $\begin{array}{l}\text { Montant TTC } \\
\left(\times 10^{3} \mathrm{~F} \mathrm{CFP}\right)\end{array}$ \\
\hline Détachement expert arboriculture fruitière & 66234 \\
\hline Fonctionnement (déplacements expert et divers) & 13556 \\
\hline Investissement (véhicule, mat. bureau, mobilier, etc .) & 8576 \\
\hline Mission d'appui & 8197 \\
\hline \multicolumn{2}{|c|}{$\begin{array}{l}\text { 2. Financement associé au projet, attribué sur contrat de développement (crédits spéciaux } \\
\text { affectés à la Polynésie par l'État français) de } 2000 \text { à } 2004: 68457 \times 10^{3} \text { F CFP TTC. }\end{array}$} \\
\hline Opérations & $\begin{array}{l}\text { Montant TTC } \\
\left(\times 10^{3} \mathrm{~F} \mathrm{CFP}\right)\end{array}$ \\
\hline Prospection et connaissance de la filière & 5010 \\
\hline Étude des systèmes d'activité des producteurs marquisiens & 1785 \\
\hline Étude des systèmes de production fruitière aux îles du Vent & 1055 \\
\hline Étude des possibilités d'approvisionnement en mangues et goyaves & 2170 \\
\hline Formation des acteurs de la filière & 28580 \\
\hline Formation des agents des conservatoires & 1190 \\
\hline Formation des agents pépiniéristes & 3868 \\
\hline Formation en arboriculture fruitière & 9891 \\
\hline Réalisation de supports techniques fruits & 7431 \\
\hline Mise en place de parcelles de démonstration & 6200 \\
\hline Amélioration des pratiques culturales & 3583 \\
\hline Analyses d'échantillons de sols & 3150 \\
\hline Production de plants fruitiers de qualité & 433 \\
\hline Amélioration du matériel végétal & 31284 \\
\hline Équipement de pépinières & 18700 \\
\hline Matériel et produits de laboratoire & 12584 \\
\hline
\end{tabular}

toutes les opérations préconisées par les expertises: équipements des pépinières, formations des agriculteurs, mise en place de conservatoires, etc. (tableau III). Cet aspect a été déterminant dans la réussite du projet.

\section{Discussion}

Le déroulement du projet d'appui aux producteurs de fruits de la Polynésie française a mis en évidence certaines particularités propres à la situation géographique et politique du Territoire, qui constituent des handicaps ou des avantages pour le développement fruitier escompté. Des enseignements intéressants peuvent être tirés de l'expérience.

\subsection{Les handicaps}

La Polynésie française est caractérisée par une très grande diversité des sols et des climats ce qui gêne une action de développement agricole. De plus, en dehors de Tahiti, il n'y a pas de passé arboricole ni de réelle profession dans ce domaine.

L'étendue et la dispersion du Territoire engendrent pour le responsable de projet beaucoup de déplacements et de perte de temps, donc des coûts importants.

Faute d'une structure de recherche agronomique structurée sur le Territoire, les différents acteurs de la filière " fruits " subissent un certain isolement scientifique. Par manque de moyens, il n'y a pas une masse 
critique de chercheurs ou techniciens spécialisés. Le recours à des missions d'appui scientifique très spécialisées ne remplace que difficilement la présence permanente de chercheurs.

Le niveau de formation des agents du SDR et des agriculteurs est en général insuffisant. En outre, avant le démarrage du projet, plusieurs responsables affectés par le SDR à la filière " fruits " s'étaient succédé au cours des années. Cela a engendré un manque de continuité dans le développement de la filière.

Des changements politiques importants et répétés de mai 2004 à février 2005 ont entraîné des retards administratifs qui ont ralenti la mise en œuvre de différentes opérations.

Les changements d'objectifs de la politique agricole imputables aux récents évènements (trois changements de ministre de l'Agriculture en 1 an) n'ont pas permis d'assurer la prolongation du projet, alors que, au sein même de la filière, nombreux étaient les acteurs du développement qui auraient souhaité poursuivre l'opération.

La relative pénurie du marché en produits n'incite guère les agriculteurs à se grouper et à commercialiser des productions de qualité. Le niveau des prix reste élevé freinant ainsi la consommation des ménages.

\subsection{Les avantages}

La présence d'un correspondant permanent du projet, responsable de la filière "fruits ", a été une formule efficace. En effet, cet agent est le relais permettant de faire appliquer les recommandations données par l'expert ; ayant pu suivre, pas à pas, la progression du projet et y ayant lui-même contribué, il sera apte à poursuivre le développement de la filière au-delà des limites fixées pour le déroulement des opérations.

Le choix d'un expert senior pour gérer le projet de développement fruitier a été également un avantage pour gérer la diversité des productions et des situations, parfois assez complexes et demandant une bonne expérience pour intégrer tous les facteurs en présence.
L'ensemble des techniciens du SDR a fait un bon accueil au projet. Ils ont exprimé un grand besoin de formation et d'encadrement. Le regain de motivation des agents qui a découlé de l'expérience a engendré d'excellents résultats dans certaines pépinières.

La possibilité de mobiliser des crédits d'État appelés " contrats de développement " a représenté un réel avantage pour le projet. Ces crédits ont permis de donner des moyens de travail à la filière "fruits " et de former les agents du SDR.

\subsection{Enseignements}

L'opération de développement des productions fruitières en Polynésie française, réalisée sur trois ans, s'est révélée trop courte. Plusieurs volets du programme n'ont pas pu être traités jusqu'au bout : ainsi, le thème "parcelles de démonstration" n'a pas été mené à son terme. D'autre part, l'aspect "structuration de la profession et amélioration des techniques post récolte " n'a été qu'effleuré. Enfin, la formation des techniciens et des agriculteurs aurait mérité d'être prolongée dans le temps par des participations actives à l'entretien de parcelles de cultures fruitières. Pour éviter la dispersion, le chef de projet s'est surtout attaché à traiter les problèmes inhérents à la culture des agrumes puisque ceux-ci représentaient la majorité des superficies fruitières. Cependant, la diversification, qui est un thème privilégié pour l'avenir, n'a pas pu être réellement abordée.

Dans le domaine de la multiplication du matériel végétal, il aurait été souhaitable que davantage de pépiniéristes privés soient formés, du moins sur les îles de la Société où il existe un réel marché pour la production de plants fruitiers de différentes espèces. Un tel développement des pépinières ne pourra vraiment s'observer que lorsque les prix pratiqués par le SDR traduiront la réalité des prix de revient, ce qui sera apte à susciter les initiatives d'opérateurs privés. La mise en place d'un système de certification des plants paraît indispensable à terme, mais cette démarche est du ressort de spécialistes qui n'existent pas localement. 
Sur le plan de la recherche d'accompagnement, il est difficilement possible de transposer, en l'état, des résultats de recherche obtenus dans d'autres situations géographiques. Une adaptation locale de tels résultats devrait être étudiée par une structure de recherche ou un institut technique. À titre d'exemple, les conservatoires variétaux existants, qui ont pu fournir des indications précieuses sur les variétés d'agrumes à diffuser, méritent maintenant d'être renouvelés. Pour cela, il faudrait qu'un travail d'observations et d'exploitation soit effectué par un chercheur compétent.

\section{Conclusion}

De nombreux aspects positifs caractérisent le projet de développement des productions fruitières réalisé en Polynésie française de 2002 à 2005. De nombreuses opérations d'appui technique, de formation, de vulgarisation, de multiplication du matériel végétal et de recherches d'accompagnement ont pu être effectuées avec succès.

Pour un développement plus harmonieux et plus durable de la filière "fruits ", et plus généralement d'autres spéculations comme le maraîchage et l'horticulture, il conviendrait de disposer sur place de structures de recherche et de développement permanentes, ainsi que de domaines expérimentaux plus importants.

\section{Remerciements}

Le " projet d'appui aux producteurs de fruits des Marquises " n'a pu se réaliser que grâce à la collaboration de nombreuses personnes qui de près ou de loin ont contribué à la mise en place et à la bonne marche de l'opération. Nous citerons le maire de Ua Huka, Léon Litchle, qui en a été l'instigateur, mais aussi tous les maires des archipels, le ministère de l'Agriculture qui a financé l'opération, l'ensemble des ingénieurs et techniciens du Service de développement rural (SDR) qui se sont impliqués avec enthousiasme dans le projet. Qu'ils en soient tous remerciés. Enfin, nous souhaitons remercier tout particulièrement Madame Sylviane Fauvet, responsable de la filière "fruits " au SDR, qui, pendant trois ans, n'a pas ménagé sa peine pour nous accompagner et pour mobiliser les crédits nécessaires à la réalisation des opérations préconisées.

\section{Références}

[1] Aubert B., État de l'agrumiculture en Polynésie française : stratégies de développement. Mission d'évaluation effectuée du 21 juillet au 7 août 1995, Rapp. interne, Cirad-Flhor, Montpellier, France, 1995.

[2] Goguey T., Ollitrault P., Suivi et évaluation du programme Agrumes en Polynésie française, Mission en Polynésie française du 13 au 30/ 10/98, Cirad-Flhor, Rapp. Interne, Montpellier, France, 1998.

[3] Anon., Bulletin de statistiques agricoles, Service du développement rural, Papeete, Polynésie française, 2003.

[4] Quilici S., État des lieux de la pression parasitaire due aux insectes sur les fruits aux Australes, aux îles du Vent et aux Marquises, Cirad-Flhor, Doc. Interne, Saint-Pierre, île de la Réunion, 2003.

[5] Grisoni M., Mu L., État des lieux des maladies des cultures fruitières en Polynésie, Mission en Polynésie française du 20/4 au 3/05/03, Cirad-Flhor, Rapp. Interne, Saint-Pierre, île de la Réunion, 2003.

[6] Jacquemond C., Curk F., Conservatoires et vergers d'agrumes en Polynésie française. Mission en Polynésie française du 22/03 au 28/04/05, Cirad-Flhor, Rapp. Interne, San Giuliano, France, 2005.

[7] Talem X., Les systèmes d'activités marquisiens et leur articulation avec Tahiti, Cnearc, Mém. Esat-2-Diat, option Valor, Montpellier, France, 2002.

[8] De La Torre C., La culture des agrumes dans les îles du Vent. Diagnostic de la situation pour des propositions d'appui technique aux producteurs, Mém. Esat-2-Diat, option Agir, Montpellier, France, 2003.

[9] Faucon G., Comment valoriser les mangues et goyaves de îles Marquises pour un industriel tel que l'usine de jus de fruits de Moorea? Istom, Mém. fin d'études, CergyPontoise, France, 2004 


\section{Análisis de un programa de desarrollo de producciones frutales en Polinesia} francesa.

Resumen - Contexto y presentación del proyecto. Tras un resumen histórico y una visión general de la arboricultura frutal en Polinesia francesa, se presentaron el funcionamiento y las operaciones programadas en 3 años de un proyecto de apoyo llevado a cabo desde 2002 hasta 2005. Realización. Los cinco temas principales de intervención se describen sucesivamente : mejora del material vegetal ; investigaciones de acompañamiento ; formación y vulgarización : tema completada por la redacción de documentos técnicos ; misiones de apoyo y períodos de prácticas ; gestión de la calidad y organización de la profesión, completado por un aspecto de valorización de los productos por transformación. Se analizaron los resultados y los medios utilizados. Discusión y conclusiones. Las ventajas e inconvenientes encontrados en la realización del proyecto se discuten y permiten proponer ciertas mejoras en la puesta en marcha de proyectos análogos.

Polinesia Francesa / fruticultura / desarrollo agrícola / encuestas / introducción de plantas / elección de especies 